\title{
ROLE OF PLEURAL BIOPSY IN THE EVALUATION OF UNDIAGNOSED EXUDATIVE PLEURAL EFFUSION
}

\author{
Bharathibabu $K^{1}$, Elamparithi $S^{2}$, Saravanavasan $R^{3}$
}

${ }^{1}$ Associate Professor, Department of Tuberculosis and Respiratory Diseases, Madurai Medical College.

${ }^{2}$ Assistant Professor, Department of Tuberculosis and Respiratory Diseases, Madurai Medical College.

${ }^{3}$ Assistant Professor, Department of Tuberculosis and Respiratory Diseases, Madurai Medical College.

\section{ABSTRACT}

\section{BACKGROUND}

Determining the cause of a pleural effusion is greatly facilitated by analysis of the pleural fluid. Many pleural effusions remain undiagnosed after routine tests of pleural fluid. So, we need a simple and safe investigative tool to evaluate such undiagnosed pleural effusions.

\section{MATERIALS AND METHODS}

Patients with undiagnosed exudative pleural effusion after pleural fluid analysis were subjected to pleural biopsy using Abram's pleural biopsy needle under local anaesthesia and the specimen was sent, histopathological examination, reverse transcriptase polymerase chain reaction for tuberculosis and culture for tuberculosis by BACTEC.

\section{RESULTS}

About $78.5 \%$ of cases were diagnosed when combining histopathological examination, BACTEC culture and reverse transcriptase polymerase chain reaction on pleural biopsy specimen.

\section{CONCLUSION}

Closed pleural biopsy can be used as an easy, quick, cost effective and relatively safe method to diagnose an exudative effusion not diagnosed by pleural fluid analysis. Combination of histopathological examination, BACTEC culture and reverse transcriptase polymerase chain reaction in closed pleural biopsy has a greater diagnostic yield in diagnosing exudative effusions not diagnosed by pleural fluid analysis.

\section{KEYWORDS}

Undiagnosed Pleural Effusion, Closed Pleural Biopsy, Abram's Needle.

HOW TO CITE THIS ARTICLE: Bharathibabu K, Elamparithi S, Saravanavasan R. Role of pleural biopsy in the evaluation of undiagnosed exudative pleural effusion. J. Evolution Med. Dent. Sci. 2017;6(65):4724-4728, DOI: 10.14260/Jemds/2017/1023

\section{BACKGROUND}

Pleural effusion is a collection of fluid in the pleural space. It is not a disease, but rather a complication of an underlying illness. Effusion can occur for a variety of reasons. Common classification systems divide pleural effusions into two categories of transudative pleural effusions and exudative pleural effusions. Determining the cause of a pleural effusion is greatly facilitated by analysis of the pleural fluid. Thoracentesis is a simple bedside procedure that permits fluid to be rapidly sampled, visualised, examined microscopically and quantified. A systematic approach to analysis of the fluid in conjunction with the clinical presentation should allow the clinician to diagnose the cause of an effusion. Pleural effusion remains undiagnosed after routine tests in pleural fluid in many patients. So, we need a simple and safe investigative tool to evaluate undiagnosed effusion. This study is designed to diagnose the cases of undiagnosed effusions by blind pleural biopsy.

Financial or Other, Competing Interest: None.

Submission 26-07-2017, Peer Review 07-08-2017,

Acceptance 09-08-2017, Published 14-08-2017.

Corresponding Author:

Dr. Elamparithi $S$,

Assistant Professor,

Department of Tuberculosis and Respiratory Diseases,

Madurai Medical College.

E-mail: solanommc@gmail.com

DOI: $10.14260 /$ jemds/2017/1023

\section{Aims and Objectives}

- To measure the additional yield of closed pleural biopsy in diagnosing exudative pleural effusions not diagnosed by pleural fluid analysis.

- To compare the yield of each test (histopathological examination, reverse transcriptase polymerase chain reaction and culture for tuberculosis by BACTEC) in pleural biopsy specimens of undiagnosed exudative effusions.

\section{MATERIALS AND METHODS}

- Study Design- Observational study.

- Study Period- June 2013 to June 2015.

- Study Centre- Government Rajaji Hospital, Madurai.

- Study Population- Patients with exudative pleural effusion remaining undiagnosed after pleural fluid analysis.

- Inclusion Criteria- Exudative effusion, which is negative for malignant cells, acid-fast bacillus, Gram stain and non-tuberculous culture in pleural fluid with ADA less than 60 .

\section{Exclusion Criteria}

- Exudative effusion positive for malignant cells.

- Exudative effusion positive for tuberculosis bacilli in pleural fluid smear.

- Exudative effusion positive for Gram stain or nontuberculous mycobacteria by culture. 
- Parenchymal lesion in the $\mathrm{x}$-ray suggestive of tuberculosis.

Malignancy or Parapneumonic Effusion

- Uncooperative patients.

- Coagulation disorders.

- Dry pleural tapping.

- Empyema.

- Uraemia.

\section{Study Procedure}

Patients with undiagnosed exudative pleural effusion, fulfilling inclusion criteria were admitted in Pulmonary Medicine Ward at Government Rajaji Hospital, Madurai. Informed and written consent obtained from the patient. Investigations were done to rule out renal diseases and coagulation abnormality. Pleural fluid sent for basic biochemical and microbiological investigations. Pleural biopsy was performed using ABRAM'S pleural biopsy needle under strict aseptic precaution under local anaesthesia and the specimen was sent for the following investigations-

- Tissue - Histopathological examination.

- Tissue - Reverse transcriptase polymerase chain reaction for tuberculosis.

- Tissue - Culture for tuberculosis by BACTEC.

\section{RESULTS}

Pleural biopsy was done in 51 patients (44 males and 7 females) and the results of histopathological examination, BACTEC culture and reverse transcriptase polymerase chain reaction on the pleural biopsy specimens were analysed and the results of which were as follows.

The data were entered in excel sheet and outcomes are measured in frequency and percentage.

\section{Age and Sex Distribution}

During the study period of 6 months, pleural biopsy was done in 51 patients of undiagnosed exudative effusions, out of which, there were 7 females and 44 males.

\begin{tabular}{|c|c|c|c|}
\hline Years & $\mathbf{1 9 - 3 5}$ yrs. & $\mathbf{3 6 - 5 0}$ yrs. & >50 yrs. \\
\hline Male & 18 & 8 & 18 \\
\hline Female & 3 & 1 & 3 \\
\hline \multicolumn{4}{|c|}{ Table 1 } \\
\hline
\end{tabular}

\section{Yield of HPE in Pleural Biopsy}

Histopathological examination diagnosed $45.1 \%$ of biopsy specimens as tuberculosis and $23.5 \%$ as malignancy. The rest $31.4 \%$ of case were left undiagnosed after histopathological examination of pleural biopsy.

\section{RT-PCR in Pleural Biopsy}

Reverse transcriptase polymerase chain reaction was positive in $17.6 \%$ of the cases and was negative in $82.4 \%$ of the cases (that is 9 out of the 51 cases were positive for RTPCR).

\section{BACTEC in Pleural Biopsy}

BACTEC was able to diagnose only 4 cases in the pleural biopsy specimens. BACTEC was positive in $7.84 \%$ of the cases and was negative in $92.16 \%$ of the cases.
Incidence of Pleural Malignancies

\begin{tabular}{|c|c|c|}
\hline HPE & Frequency (n=12) & Percent \\
\hline Adenocarcinoma & 6 & $50 \%$ \\
\hline Squamous cell carcinoma & 4 & $33.33 \%$ \\
\hline Lymphoma & 1 & $8.33 \%$ \\
\hline Small cell carcinoma & 1 & $8.33 \%$ \\
\hline \multicolumn{2}{|c}{ Table 2 } \\
\hline
\end{tabular}

Yield of Pleural Biopsy

\begin{tabular}{|c|c|c|c|c|}
\hline & Results & $\mathbf{1 9 - 3 5}$ yrs. & $\mathbf{3 5 - 5 0}$ yrs. & $\mathbf{> 5 0}$ yrs. \\
\hline \multirow{3}{*}{ Male } & Tuberculosis & 16 & 4 & 4 \\
\cline { 2 - 5 } & Malignancy & 0 & 1 & 9 \\
\cline { 2 - 5 } & Undiagnosed & 2 & 3 & 5 \\
\hline \multirow{3}{*}{ Female } & Tuberculosis & 2 & 1 & 1 \\
\cline { 2 - 5 } & Malignancy & 0 & 0 & 2 \\
\cline { 2 - 5 } & Undiagnosed & 1 & 0 & 0 \\
\hline Total & \multicolumn{4}{|c|}{ Table 3 } \\
\hline \multicolumn{5}{|c|}{$\mathbf{9 1}$} \\
\hline
\end{tabular}

\section{Cases Diagnosed by Pleural Biopsy}

\begin{tabular}{|c|c|c|}
\hline $\begin{array}{c}\text { Combining HPE, } \\
\text { BACTEC AND RT-PCR }\end{array}$ & Frequency (n=51) & Percent \% \\
\hline Diagnosed & 40 & $78.5 \%$ \\
\hline Undiagnosed & 11 & $21.5 \%$ \\
\hline \multicolumn{3}{|c|}{ Table 4 } \\
\hline
\end{tabular}

About $78.5 \%$ of cases were diagnosed when combining histopathological examination, BACTEC culture and reverse transcriptase polymerase chain reaction on pleural biopsy specimen. $21.5 \%$ of cases were left undiagnosed even after combining all the three tests.

\section{Follow up}

Patients were followed up for a period of 6 months after pleural biopsy. All patients diagnosed as TB pleural effusion had excellent response to ATT. Patients with malignant pleural effusion were sent to Medical Oncology Department for further management. Five out of the eleven patients left undiagnosed after pleural biopsy were found to have malignancies in follow up.

\section{DISCUSSION}

Pleural fluid accumulates when pleural fluid formation exceeds pleural fluid absorption. Normally, fluid enters the pleural space from the capillaries in the parietal pleura and is removed via the lymphatics situated in the parietal pleura. Fluid can also enter the pleural space from the interstitial spaces of the lung via the visceral pleura or from the peritoneal cavity via small holes in the diaphragm. The lymphatics have the capacity to absorb 20 times more fluid than is normally formed. Accordingly, a pleural effusion may develop when there is excess pleural fluid formation (from the interstitial spaces of the lung, the parietal pleura or the peritoneal cavity) or when there is decreased fluid removal by the lymphatics. When a patient is found to have a pleural effusion, an effort should be made to determine the cause. The first step is to determine whether the effusion is a transudate or an exudate. A transudative pleural effusion occurs when systemic factors that influence the formation and absorption of pleural fluid are altered. The leading causes of transudative pleural effusions are left ventricular 
failure and cirrhosis. An exudative pleural effusion occurs when local factors that influence the formation and absorption of pleural fluid are altered. The leading causes of exudative pleural effusions are tuberculosis, bacterial pneumonia, malignance, viral infection and pulmonary embolism. The primary reason to make this differentiation is that additional diagnostic procedures are indicated with exudative effusions to define the cause of the local disease.

\section{Pleural Biopsy}

Pleural biopsy is helpful to reach a diagnosis in exudative pleural effusion, particularly when the results of detailed pleural fluid study are inconclusive especially in a setup where thoracoscopy is not available. The main contraindication to a pleural biopsy is a bleeding diathesis, empyema,(1) uncooperative patient and local cutaneous lesions such as pyoderma or herpes zoster infection at the planned site of pleural biopsy. When there is a moderate or larger pleural effusion, the biopsy is usually done without image guidance. If the effusion is small or loculated, then ultrasound is used to locate the site. Pleural biopsy is usually performed with an Abram's needle. Pneumothorax is the most common complication of closed pleural biopsy. However, the incidence of pneumothorax and the requirement for tube thoracostomy are comparable after thoracentesis and pleural biopsy.(2) Bleeding is the second most common complication reported. If an intercostals artery or vein is inadvertently biopsied, a haemothorax can result.(3) The percentage of positive pleural biopsies in patients with malignant pleural disease ranges from $39 \%$ to $75 \% \cdot{ }^{(4,5,6)}$

\section{Indications of Pleural Biopsy}

A needle biopsy of the pleura is currently recommended when tuberculous pleuritis is suspected and the pleural fluid ADA or interferon-gamma levels are not definitive. A needle biopsy of the pleura is also recommended when malignancy is suspected, but the pleural fluid cytology is negative and thoracoscopy is not readily available. When a needle biopsy of the pleura, a small piece of the parietal pleura is obtained for microscopic or microbiologic evaluation. The main diagnoses established with a needle biopsy of the pleura are tuberculous pleuritis and malignancy of the pleura. Currently, needle biopsy of the pleura is used less than in the past because the diagnosis of tuberculous pleuritis can be made by measuring the Adenosine Deaminase (ADA) or interferon-gamma level in the pleural fluid and the diagnosis of pleural malignancy is usually established by pleural fluid cytology or thoracoscopy.

\section{Contraindications of Pleural Biopsy}

The main contraindication to a pleural biopsy is a bleeding diathesis. Another contraindication to needle biopsies is the presence of an empyema. Other contraindications include an uncooperative patient and local cutaneous lesions such as pyoderma or herpes zoster infection. A pleural biopsy should not be performed in patients who are taking anticoagulants or whose bleeding parameters are prolonged. If the platelet count is below $50,000 / \mathrm{mm} 3$, platelet transfusion should be given before the procedure is attempted. If the patient has borderline respiratory failure, one should hesitate to perform a pleural biopsy because the production of pneumothorax could precipitate respiratory failure.

\begin{abstract}
Abram's Needle
The Abram's needle consists of three parts- A large outer trocar, an inner cutting cannula and an inner solid stylet. The end of the outer trocar is blunt so that the instrument will not lacerate the lung, but the bluntness of the instrument requires one to make a small scalpel incision in the anaesthetised skin and subcutaneous tissue to permit insertion of the biopsy needle without undue force. This incision should be made along the lines of cleavage to minimise postoperative scarring. The inner cutting cannula fits tightly in the outer trocar and can be locked in one of two positions- (a) A closed position, in which the inner cannula obstructs the notch on the outer trocar to make the needle airtight and (b) An open position, in which the inner cannula is slightly withdrawn, so that the notch on the outer trocar is not occluded. An indicator knob in the hexagonal grip of the larger outer trocar indicates the position of the notch in the distal end of the trocar.
\end{abstract}

\section{Method of Pleural Biopsy}

The patient was positioned and the site was selected either clinically or by image guidance. The skin was cleaned and the local anaesthetic was administered as for diagnostic thoracentesis. Liberal amount of lignocaine was injected once the rib is passed to ensure adequate anaesthesia of the parietal pleura. Skin incision was made at the selected site to insert the Abram's pleural biopsy needle. The stylet was placed in the inner cannula, which in turn was placed in the outer trocar. The inner cannula was twisted clockwise to close the distal notch of the outer trocar. The needle was pushed into the pleural space by exerting firm pressure on the stylet. Because the needle has a large diameter and is blunt, a substantial amount of pressure was needed. Usually, a pop was heard as the needle enters the pleural space. The inability to pass the needle into the pleural space is usually because of an insufficiently large skin incision. At times, the ribs were too close together to allow the needle to pass. In such situations, rotation of the patient's arm and shoulder over his or her head frequently separated the ribs sufficiently. Once the tip of the needle is thought to be in the pleural space, the inner stylet was removed and with the inner cannula in the closed position. A syringe was attached to the connection on the inner cannula. Then, the inner cannula was rotated counter-clockwise in the outer trocar, so that the distal notch was locked upon. At this time, pleural fluid was aspirated for diagnostic studies. When the desired fluid has been obtained, the inner cannula of the needle was rotated clockwise to occlude the distal notch, so that the syringe can be changed without creating a pneumothorax. A 10 - to $20-\mathrm{mL}$ syringe was then attached to the needle and the inner cannula was rotated to open the distal notch. The entire needle was then rotated, so that the knob on the outer trocar was inferior. This was important so that the blood vessels and nerves that lie immediately below the rib were not biopsied. The biopsy needle was then slowly withdrawn with constant aspiration until it hooked onto the pleura. When the needle hooks, one can be sure that parietal pleura was in the notch of the needle if pleural fluid can still be aspirated through the syringe. When the needle was hooked 
on the pleura, the outer trocar was held firmly with one hand, while the inner cannula was rotated into the closed position with the other hand to cut off a small piece of parietal pleura. Usually, mild resistance was met immediately before the needle was completely closed and this resistance is because the inner cannula severing the entrapped pleura for the biopsy specimen. Once the initial biopsy specimen was obtained, the needle can either be withdrawn from the pleural space in the closed position. The pleural biopsy specimen was found in the tip of the needle. Reinsertions of the needle are through the same tract, however, and are easier than the original insertion. The biopsy procedure can be repeated without removing the biopsy needle. Whenever the Abram's pleural biopsy needle was withdrawn from the pleural space, the biopsy tract was occluded with a finger immediately after the needle was withdrawn to decrease the likelihood of a pneumothorax. At least, six separate biopsy specimens were obtained. Four were placed in formalin and were taken to the pathology laboratory and the other two were placed in a sterile container and sent to the laboratory for BACTEC culture and reverse transcriptase polymerase chain reaction targeting $85 \mathrm{~B}$ gene of mycobacterium tuberculosis. Once the biopsy specimens were obtained, a therapeutic thoracentesis was performed through the Abram's needle. The pleural fluid should be removed only after obtaining the biopsy specimens because the pleural fluid separates the parietal and visceral pleura and increases the safety of the procedure. When the Abram's needle was withdrawn for the last time, the biopsy site was massaged for a short time to eradicate the needle tract. Then, a small adhesive bandage was placed over the biopsy incision in a crosswise manner to act as a butterfly-type dressing. Chest radiographs were obtained on all patients after pleural biopsies.

\section{Reverse Transcriptase Polymerase Chain Reaction}

PCR is a technique to amplify a single or few copies of a piece of DNA across several orders of magnitude generating millions or more copies of a particular DNA sequence. RTPCR detects m-RNA of the organism under study. RT-PCR targeting $85 \mathrm{~B}$ gene is very specific for mycobacterium tuberculosis and was used in our study. It is negative for non-tuberculous mycobacterium. mRNA detected by RT-PCR has a half-life for only few minutes and can detect only viable mycobacterium.

\section{BACTEC Culture}

The rapid radiometric culture system has been accepted for the culture isolation of mycobacteria using an enriched Middlebrook 7H12 containing 14C labeled palmitic acid. This medium is otherwise called BACTEC 12B. In comparison to the conventional $\mathrm{M}$. tuberculosis culture using Lowenstein-Jensen Media, the BACTEC system gives early culture results with differentiation of M. tuberculosis from mycobacteria other than M. tuberculosis. $(7,8,9,10)$

\section{Clinical Implication}

Needle biopsy of the pleura can establish the diagnosis of a malignant pleural effusion. The percentage of positive pleural biopsies in patients with malignant pleural disease ranges from $39 \%$ to $75 \%$. In general, pleural fluid cytology is superior to pleural biopsy in establishing the diagnosis of pleural malignancy. The diagnostic yield of pleural biopsy in our study was $70 \%$ in malignant cases. In one study of 248 patients with tuberculous pleuritis who underwent needle biopsy of the pleura, the biopsy showed granulomas in 198 patients (80\%), the acid-fast stain of the biopsy was positive in $64(25.8 \%)$, the culture of the biopsy tissue was positive in $140(56 \%)$ and at least one of the preceding three tests was positive in 227 (91\%). Polymerase chain reaction has also been tried on pleural biopsy specimens, but it is not clear that the polymerase chain reaction adds to the regular microscopic examination. In our study, histopathological examination diagnosed $67 \%$ of tuberculous effusion. Reverse transcriptase polymerase chain reaction diagnosed $26.1 \%$ of tuberculous effusion. BACTEC diagnosed $11.8 \%$ of tuberculous effusion. $82.3 \%$ of tuberculous cases were diagnosed by combining all three tests. As there were no false-positive results with any of the three tests (histopathological examination, reverse transcriptase polymerase chain reaction and BACTEC culture), they are highly specific in diagnosing both tuberculosis and malignancy. Both the specificity and positive predictive value were $100 \%$ with these tests. As these tests are highly specific, we can start treatment even when any of these test is positive.

\section{CONCLUSION}

- Combination of histopathological examination, BACTEC culture and reverse transcriptase polymerase chain reaction in closed pleural biopsy has a greater diagnostic yield in diagnosing exudative effusions not diagnosed by pleural fluid analysis.

- Histopathological examination diagnosed $67 \%$ of tuberculous effusion.

- Reverse transcriptase polymerase chain reaction diagnosed $26.1 \%$ of tuberculous effusion.

- BACTEC diagnosed $11.8 \%$ of tuberculous effusion.

- Pleural biopsy diagnosed 79\% of undiagnosed effusion.

- Closed pleural biopsy can be used as an easy, quick, cost effective and relatively safe method to diagnose an exudative effusion not diagnosed by pleural fluid analysis.

\section{REFERENCES}

[1] Levine H, Cugell DW. Blunt-end needle biopsy of pleura and rib. Arch Intern Med 1971;109:516-25.

[2] Poe RH, Israel RH, Utell MJ, et al. Sensitivity, specificity, and predictive values of closed pleural biopsy. Arch Intern Med 1984;144(2):325-8.

[3] Ali J, Summer WR. Hemothorax and hyperkalemia after pleural biopsy in a 43-year-old woman on hemodialysis. Chest 1994;106(4):1235-6.

[4] Prakash UB, Reiman HM. Comparison of needle biopsy with cytologic analysis for the evaluation of pleural effusion: analysis of 414 cases. Mayo Clin Proc 1985;60(3):158-64.

[5] Sayler WR, Eggleston JC, Erozan YS. Efficacy of pleural needle biopsy and pleural fluid cytopathology in the diagnosis of malignant neoplasm involving the pleura. Chest 1975;67(5):536-9. 
[6] Frist B, Kahan AV, Koss LG. Comparisons of the diagnostic values of biopsies of the pleura and cytologic evaluation of pleural fluids. Am J Clin Pathol 1979;72(1):48-51.

[7] Park CH, Hixon DL, Ferguson CB, et al. Rapid recovery of mycobacteria from clinical specimens using automated radiometric technic. Am J Clin Path 1984;81(3):341-5.
[8] Takahasi H, Foster V. Detection and recovery of mycobacteria by a radiometric procedure. J Clin Microbiol 1983;17(2):380-1.

[9] Laszlo A, Handzel V. Radiometric diagnosis of mycobacteria. Eur J Clin Microbiol 1986;5(2):152-5.

[10] Laglo A, Michaud R. Primary isolation, preliminary identification and drug susceptibility testing of mycobacteria by a rapid radiometric method. Bull IUAT 1984;59:185-7. 